\title{
Sialyltransferase Regulates Nervous System Function in Drosophila
}

\author{
Elena Repnikova, ${ }^{1 \star}$ Kate Koles, ${ }^{1 \star}$ Michiko Nakamura, ${ }^{1}$ Jared Pitts, ${ }^{1}$ Haiwen Li, ${ }^{1}$ Apoorva Ambavane, ${ }^{1}$ Mark J. Zoran, ${ }^{2}$ \\ and Vladislav M. Panin ${ }^{1}$ \\ Departments of ${ }^{1}$ Biochemistry and Biophysics and ${ }^{2}$ Biology, Texas A\&M University, College Station, Texas 77843
}

In vertebrates, sialylated glycans participate in a wide range of biological processes and affect the development and function of the nervous system. While the complexity of glycosylation and the functional redundancy among sialyltransferases provide obstacles for revealing biological roles of sialylation in mammals, Drosophila possesses a sole vertebrate-type sialyltransferase, Drosophila sialyltransferase (DSiaT), with significant homology to its mammalian counterparts, suggesting that Drosophila could be a suitable model to investigate the function of sialylation. To explore this possibility and investigate the role of sialylation in Drosophila, we inactivated DSiaT in vivo by gene targeting and analyzed phenotypes of DSiaT mutants using a combination of behavioral, immunolabeling, electrophysiological, and pharmacological approaches. Our experiments demonstrated that DSiaT expression is restricted to a subset of CNS neurons throughout development. We found that DSiaT mutations result in significantly decreased life span, locomotor abnormalities, temperature-sensitive paralysis, and defects of neuromuscular junctions. Our results indicate that DSiaT regulates neuronal excitability and affects the function of a voltage-gated sodium channel. Finally, we showed that sialyltransferase activity is required for DSiaT function in vivo, which suggests that DSiaT mutant phenotypes result from a defect in sialylation of N-glycans. This work provided the first evidence that sialylation has an important biological function in protostomes, while also revealing a novel, nervous system-specific function of $\alpha 2,6$-sialylation. Thus, our data shed light on one of the most ancient functions of sialic acids in metazoan organisms and suggest a possibility that this function is evolutionarily conserved between flies and mammals.

\section{Introduction}

Sialic acids, a family of nine-carbon backbone acidic sugars, mainly occupy terminal positions of carbohydrate modification of glycoproteins and glycolipids (Angata and Varki, 2002). In vertebrates, sialylated glycans are abundantly present on cell surfaces and in the extracellular milieu; they are involved in cell adhesion and cell communication, participating in a variety of crucial biological processes (Varki, 2007). Abnormal sialylation has been implicated in a number of pathobiological conditions, including neurological diseases, immunodeficiency, and cancer (Varki, 2008). Glycoprotein sialylation has been extensively studied in mammals and some roles of this posttranslational modification have been determined. These roles often depend on the

\footnotetext{
Received 0ct. 20, 2009; revised Feb. 17, 2010; accepted March 23, 2010.

This work was supported by National Institute of General Medical Sciences Grant GM069952 (V.M.P.). We thank P. Hardin for comments on this manuscript, B. Ganetzky for para mutant strains, D. Featherstone for RKKeve-GAL4 flies, J. Hirsh for DDC-GAL4 driver, R. Ordway for cac ${ }^{\text {ts2 }}$ flies, L. Hall for tipE mutants, J. Dubnau for isogenized $w^{1718}$ Canton-S flies, A. DiAntonio for DVGlut antibody, J. Sisson for Lva antibody, S. Vitha for technical advice, and N. Nakamura and D. Lyalin for discussion and technical help. We acknowledge the use of Texas A\&M University Microscopy and Imaging Center confocal facility, antibodies from Developmental Studies Hybridoma Bank (University of lowa, lowa City, IA), and Drosophila strains from the Bloomington Stock Center (Indiana University, Bloomington, IN).

*E.R. and K.K. contributed approximately equally to this work.

Correspondence should be addressed to Dr. Vladislav M. Panin, Department of Biochemistry, Texas A\&M University, 2128 TAMU, College Station, TX 77843. E-mail: panin@tamu.edu.

K. Koles's present address: Neurobiology Department, University of Massachusetts Medical School, Worcester, MA 01605.

D0I:10.1523/JNEUROSC1.5253-09.2010

Copyright $\odot 2010$ the authors $\quad 0270-6474 / 10 / 306466-11 \$ 15.00 / 0$
}

nature of acceptors, as well as on the linkage of sialylation, both determined by properties of sialyltransferases, enzymes that attach sialic acid to specific acceptors (Harduin-Lepers et al., 2001). Phenotype analysis of mouse knock-outs for genes involved in sialylation has been an important approach in revealing biological functions of sialylated structures (Hennet et al., 1998; Eckhardt et al., 2000; Moody et al., 2001; Angata et al., 2004). Although the prominent role of polysialylation of the NCAM (neural cell adhesion molecule) in mammalian brain development and neurophysiology has been established (Rutishauser, 2008; Mühlenhoff et al., 2009), the function of other types of glycoprotein sialylation in the nervous system remains mostly unknown. The complexity of the mammalian nervous system, functional redundancy among sialyltransferases, and intricate regulation of sialylation pathway impede the analysis of neural functions of sialylation in mammals (Harduin-Lepers et al., 2001; Martin et al., 2002; Hildebrandt et al., 2007), suggesting that a suitable model organism could be a useful tool for this analysis. Yet, little is known about sialylation in animals besides vertebrates. Recently, Drosophila has been shown to possess functional homologues of vertebrate enzymes for several key steps in sialylation pathways, including sialic acid phosphate synthetase (Kim et al., 2002), CMP-sialic acid synthetase (Viswanathan et al., 2006), and a sialyltransferase, Drosophila sialyltransferase (DSiaT) (Koles et al., 2004). Functional characterization of DSiaT revealed its evolutionary relationship to mammalian ST6Gal sialyltransferases (Fig. 1), suggested that DSiaT functions in the nervous system, and predicted that $\mathrm{N}$-linked glycans are putative 


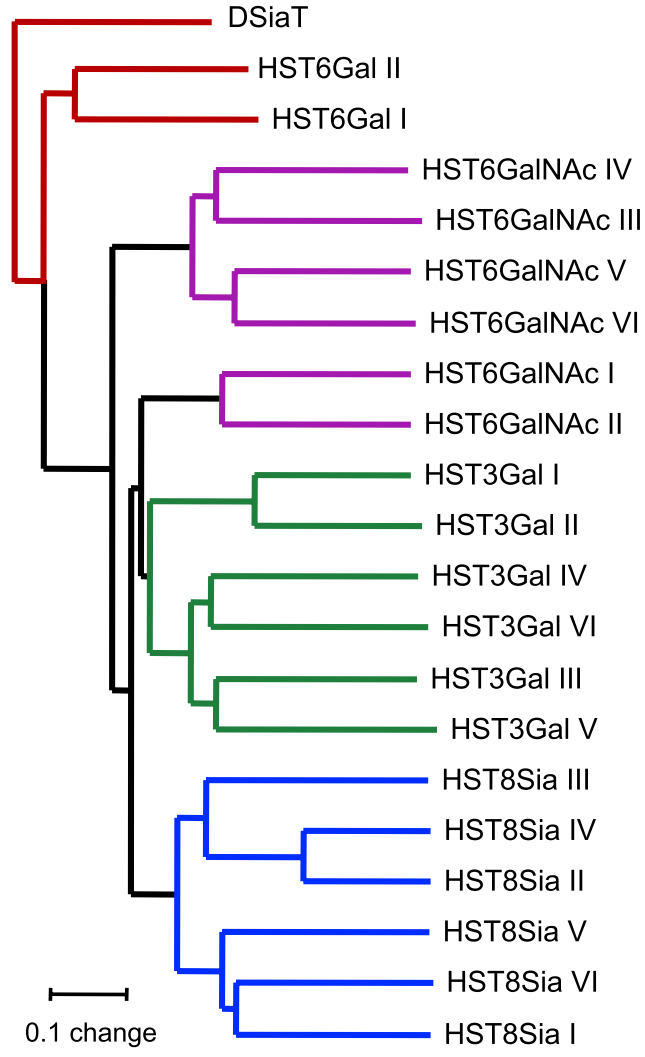

Figure 1. Phylogenetic relationship between Drosophila and human sialyltransferases. DSiaT is closely related to ST6Gal family of mammalian sialyltransferases. Sialyltransferase subfamilies with distinct substrate/linkage specificities are indicated by different colors: DSiaT and ST6Gal enzymes, red; ST6GalNAc enzymes, magenta; ST3Gal enzymes, green; ST8Sia enzymes, blue. The phylogenetic tree was constructed by Clustal W2 program at EMBL-EBI site (http:// www.ebi.ac.uk/Tools/clustalw2/index.html) (Larkin et al., 2007) using neighbor-joining method. The accession numbers of protein sequences are as follows: NP_523853 (DSiaT), NM_173216 (HST6Gal I), XM_038616 (HST6Gal II), NP_060884 (HST6GaINAC I), NP_006447 (HST6GalNAc II), NP_694541 (HST6GalNAc III), NP_778204 (HST6GaINAc IV), NP_112227 (HST6GaINAc V), Q969X2 (HST6GaINAc VI), NP_003024 (HST3Gal I), Q16842 (HST3Gal II), NP_777631 (HST3Gal III), NP_006269 (HST3Gal IV), NP_003887 (HST3Gal V), NP_006091 (HST3Gal VI), NP_003025 (HST8Sia I), NP_006002 (HST8Sia II), NP_056963 (HST8Sia III), NP_005659 (HST8Sia IV), NP_037437 (HST8Sia V), and NP_001004470 (HST8Sia VI).

targets of sialylation in vivo (Koles et al., 2004). The presence of predicted $\alpha 2,6$-sialylated N-linked glycans has been recently confirmed in Drosophila by mass spectrometry (Aoki et al., 2007; Koles et al., 2007). However, until now the biological function of sialylation in Drosophila or any other protostome species (including arthropods, annelids, and mollusks) was unknown.

To shed light on this function and analyze its relationship to the role of sialylation in higher animals, we generated Drosophila sialyltransferase knock-out mutants and analyzed their phenotypes using behavioral, genetic, electrophysiological, and pharmacological approaches. We found that DSiaT plays a pivotal role in the nervous system, regulating excitability of neurons, affecting development of neuromuscular junctions (NMJs), and influencing behaviors. Our results demonstrate that sialyltransferase enzymatic activity is required for DSiaT in vivo function and suggest that DSiaT modulates the function of voltage-gated sodium channels. Together, our results reveal a novel, neuronspecific function of ST6Gal-type sialyltransferases and suggest a possibility that this function is evolutionarily conserved in animals.

\section{Materials and Methods}

Drosophila strains

RKKeve-GAL4 was obtained from David Featherstone (University of Illinois at Chicago, Chicago, IL) (Featherstone et al., 2000). Wild-type control $w^{1118}$ Canton-S was from Josh Dubnau (Cold Spring Harbor Laboratory, Cold Spring Harbor, NY) (Dubnau et al., 2001); para ${ }^{L K 5}$ (loss-of-function allele), para $^{t s l}$ (hypomorph, conditional temperaturesensitive allele), and para ${ }^{D p}$ (aka $D p(1 ; 4) r^{+} f^{+}$, a duplication of para on the fourth chromosome) were from Barry Ganetzky (University of Wisconsin-Madison, Madison, WI) (Ganetzky, 1984); tipE $E^{1}$ (loss-offunction paralytic allele) was obtained from Linda Hall (University of California-Davis, Davis, CA) (Feng et al., 1995), and $\operatorname{cac}^{t s 2}$ (temperaturesensitive paralytic mutant) was received from Richard Ordway (Penn State University, University Park, PA) (Brooks et al., 2003). $y^{I} w^{1}$, C380GAL4, Cha-GAL4, UAS-GFP and UAS-CD8-GFP lines were from the Bloomington Stock Center (Indiana University). All Drosophila strains were reared in a controlled environment incubator $\left(25^{\circ} \mathrm{C}, 35 \%\right.$ humidity, $12 \mathrm{~h}$ light/darkness) on standard cornmeal-malt-yeast medium.

\section{Generation of DSiaT alleles}

Two loss-of-function alleles were created by homologous recombination. The S23 loss-of-function allele was generated by an ends-in genetargeting approach using pTV2 vector-based donor construct (Rong and Golic, 2000) including $8 \mathrm{~kb}$ DSiaT genomic region. S23 includes two premature stop codons within the DSiaT coding region that are predicted to inactivate the gene. The upstream stop codon is expected to prematurely terminate translation after the first 17 aa of the DSiaT protein. The downstream stop codon is predicted to result in DSiaT truncated in the middle of its S-sialylmotif and missing $84 \mathrm{C}$-terminal amino acids. Both engineered stop codons also introduced additional restriction sites (BspHI and NheI), which facilitated the analysis of the mutant (supplemental Fig. S1, available at www.jneurosci.org as supplemental material). Another loss-of-function allele, L22, was generated with an ends-out targeting technique (Gong and Golic, 2003) by introducing a deletion that removes most of the DSiaT coding sequence. Targeting was performed essentially as previously described (Gong and Golic, 2004). Briefly, the donor construct was based on pW25 vector and included $8 \mathrm{~kb}$ genomic region of DSiaT with engineered $1.7 \mathrm{~kb}$ deletion of the $2 \mathrm{~kb}$ $D S i a T$ open reading frame (ORF). The donor construct also included a 729 bp fragment encoding green fluorescent protein (GFP), so the resulting mutant ORF encoded a fusion of first 44 aa of DSiaT with GFP. The design was aimed at creating a mutant allele with GFP expression in endogenous DSiaT pattern; however, the GFP-coding sequence was damaged by a short insertion ( $\sim 30 \mathrm{bp})$ during the targeting event, which abolished GFP expression. The mini-white marker associated with donor construct was removed from targeted locus by Cre recombinase (Gong and Golic, 2004). The KI48 allele was generated using the same strategy that was applied for creating S23. KI48 includes a short sequence encoding two tandem hemagglutinin (HA) tags (Niman et al., 1983) in frame with endogenous ORF of DSiaT, which results in the expression of the DSiaT protein with two HA tags within its stem region. All three alleles were confirmed by Southern blot hybridization and sequencing of DSiaT coding region plus adjacent $2 \mathrm{~kb}$ flanking genomic fragments (supplemental Fig. S1, available at www.jneurosci.org as supplemental material).

To minimize potential influence of genetic background, DSiaT mutant alleles were outcrossed at least 10 times to their matching "wildtype" control genotypes, $w^{1118}$ Canton $S$ (designated as WT) and $y^{1} w^{1}$ (designated as $y w$ ) for $S 23$ and $L 22$ alleles, respectively. In all functional assays, S23 and L22 alleles were indistinguishable from each other, and they were used in this work interchangeably. The $P\left[D_{S i a T}{ }^{+}\right]$transgenic insertion on the third chromosome was generated by P-elementmediated transformation. The construct contained $8 \mathrm{~kb}$ genomic locus of $D S i a T$ including $\sim 3 \mathrm{~kb}$ upstream and downstream regions of the gene and no other predicted ORFs.

\section{Behavioral assays}

Larvae. For larval locomotion assays, we used larvae from controlleddensity populations (Stewart and McLean, 2004). To this end, adult parent flies were allowed to lay eggs on a grape juice plate for $3 \mathrm{~h}$. Twenty-four 
hours later, first-instar larvae were transferred from the plate to vials with $\sim 7 \mathrm{ml}$ of food, 20 larvae per vial, and allowed to develop until mid-third instar (80-84 h after egg laying). Before analyses, individual larvae were briefly rinsed in $25 \%$ sucrose and then in distilled water. Using a brush, larvae were placed gently in the center of a $100 \mathrm{~mm}, 3 \%$ agar plate. Each larva was given $2 \mathrm{~min}$ to adapt to the plate. The plate was placed onto a $0.5 \times 0.5 \mathrm{~mm}$ grid paper, and movies were recorded for $30 \mathrm{~s}$ for each larva using Sony HD digital camera. Speed and number of waves of body muscle contractions per second were obtained from straight-path crawling intervals. Distance traveled per one wave of body muscle contraction ("distance per muscle contraction") was calculated as larva speed divided by contraction frequency. For crawling pattern assay, the motion of individual third-instar larvae on agar plates was recorded during $30 \mathrm{~s}$, and digitized tracks were obtained from recorded movies by tracing larval head movements.

Adult flies. For individual longevity, adult male flies were collected within $24 \mathrm{~h}$ after eclosion. Each fly was placed into individual vial with food and transferred to new vial with fresh food every $3 \mathrm{~d}$. Group longevity was assayed with groups of 10 flies (males)/vial. Flies were transferred to fresh food every $3 \mathrm{~d}$, and dead flies were counted every day. For locomotion assays, individual flies were collected on the day of enclosure and aged for 3, 5, and $7 \mathrm{~d}$, as indicated. Before the assay, each fly was placed in an empty vial and allowed to adapt for $10 \mathrm{~min}$. Locomotion assays were performed essentially as described previously (Haines and Stewart, 2007). Briefly, the vial with a single fly was banged five times onto a soft rubber pad. The time that it took the fly to right itself after falling on its back was recorded. For each fly, the assay was performed with two trials and 10 min recovery in between. For the temperaturesensitive (TS)-paralysis assay, flies were transferred to empty vials and temperature was shifted to $38^{\circ} \mathrm{C}$ by submerging vials in a controlledtemperature water bath. We defined paralysis as a condition when a fly lies on the bottom of a vial and is unable to stand and walk. To analyze the kinetics of paralysis, flies were collected on the day of enclosure and aged as indicated. Paralysis was assayed in groups of 10 flies in empty plastic vials while counting the number of paralyzed flies in 1 min intervals. For genetic interactions, flies were assayed individually, and the time of paralysis onset was recorded for every fly.

\section{NMJ analysis}

We used third-instar larvae collected from controlled-density populations. Larvae were dissected along the ventral midline in ice-cold PBS, fixed in $4 \%$ paraformaldehyde, $50 \mathrm{~mm} \mathrm{NaCl}, 0.1 \mathrm{M}$ PIPES, pH 7.2, for 10 min at room temperature, and then their NMJs were analyzed by immunostaining. Fluorescent $z$-stack optical sections of muscle 1 from third abdominal segments were used to analyze the number of boutons, branches, and active zones. Since identified DSiaT-expressing motoneuron (MN1-Ib) produces type Ib boutons, we did not include type II boutons in our analyses. However, we counted type Ib and type Is boutons together because morphological difference between them is sometimes ambiguous. For active zone analysis, muscle 1 NMJs were double stained with rabbit anti-HRP and mouse nc 82 (anti-Bruchpilot) primary antibodies and anti-rabbit Alexa 488 and anti-mouse Cy3-conjugated secondary antibodies. Distinct nc82 puncta were counted in optical sections and verified with three-dimensional reconstructed images of individual boutons using AxioVision software. For nc82 puncta analysis, five Ib boutons (3-6 $\mu \mathrm{m}$ in size) were randomly selected from each analyzed muscle $1 \mathrm{NMJ}$ in abdominal segment 3 of late third-instar larvae.

\section{Immunofluorescent staining and microscopy}

Immunostaining was performed essentially as described previously (Lyalin et al., 2006). The following primary antibodies and corresponding dilutions were used: rabbit anti-Lva (a gift from John Sisson, University of Texas, Austin, TX), 1:2000, was used to label the Golgi compartment (Sisson et al., 2000); rabbit anti-DVGlut (a gift from Aaron DiAntonio, Washington University, St. Louis, MO), 1:4000, was used to label motor neurons (Daniels et al., 2008); rat anti-HA (Roche), 1:800, was used to reveal the pattern of DSiaT expression in KI48 allele; rabbit anti-HRP (Jackson ImmunoResearch Laboratories), 1:800, and mouse anti-Dlg (Developmental Studies Hybridoma Bank), 1:200, were used to visualize presynaptic membrane (Jan and Jan, 1982) and subsynaptic reticulum (Budnik et al., 1996), respectively. We used mouse anti-Repo antibody (1:5) to label glial cells (Alfonso and Jones, 2002). Mouse nc82 antibody from Developmental Studies Hybridoma Bank was used at 1:50 dilution to visualize adult brain neuropil and to label active zones in synaptic boutons of larval NMJs. We used the following fluorescent secondary antibodies (with corresponding dilutions): anti-mouse-Cy3 (1:250), anti-rabbit-FITC (1:150), anti-mouse-Cy5 (1:250) (all from donkey; Jackson ImmunoResearch Laboratories), and goat anti-rabbit Alexa-488 (1:140) (Invitrogen). Fluorescent images were acquired using an Olympus FV1000 confocal microscope or a Zeiss Axioplan 2 microscope with ApoTome module for optical sectioning. $Z$-projections were generated using Zeiss AxioVision and ImageJ software (Abramoff et al., 2004).

\section{Electrophysiology}

Current-clamp intracellular recordings were performed from muscles of dissected third-instar larvae essentially as previously described (Stewart et al., 1994). Briefly, Drosophila third-instar larvae were dissected in icecold $\mathrm{Ca}^{2+}$-free HL3 solution, and recordings were performed at room temperature. The recording solution was supplemented with $\mathrm{CaCl}_{2}$ as indicated for the specific experiments. We used microelectrodes with an input resistance of $8-16 \mathrm{M} \Omega$ prepared from $1.2 \mathrm{~mm}$ borosilicate glass capillaries and filled with $3 \mathrm{~m} \mathrm{KCl}$. Membrane potentials were amplified by the Axoclamp 200B amplifier (Molecular Devices), filtered at $10 \mathrm{kHz}$, digitized, and recorded on a Dell PC computer equipped with pClamp10 software (Molecular Devices). For spontaneous miniature excitatory junction potential (mini-EJP) recordings, the extracellular $\mathrm{Ca}^{2+}$ was 1 mM. Mini-EJP was analyzed using a semiautomated protocol with the event detection mode in PCLAMP and a template obtained from miniEJP recording of wild-type control genotype based on $>200$ individual events. To elicit postsynaptic response, segmental nerves were stimulated with $0.2 \mathrm{~ms}$ pulses at 2 times the stimulus amplitude required for a threshold response. Excitatory junction potentials (EJPs) were analyzed using the cursor options of Clampfit 10.0 (Molecular Devices). EJP analysis was performed by averaging 10 events for each muscle and considering the average amplitude as one measurement for the genotype assayed. Paired-pulse facilitation was performed in $0.75 \mathrm{mM} \mathrm{Ca}^{2+}$ HL3 saline essentially as described previously (Wairkar et al., 2008), with 50 $\mathrm{ms}$ period between stimulating pulses. Facilitation index was calculated as the ratio between EJP amplitudes evoked by the second and the first pulses. High-frequency stimulation was performed in $4 \mathrm{mM} \mathrm{Ca}^{2+} \mathrm{HL} 3-$ saline as described previously (Song et al., 2002; Verstreken et al., 2002) with 3000 pulses delivered to stimulated nerves at a frequency of $10 \mathrm{~Hz}$. For tetrodotoxin (TTX) sensitivity assays, $10 \mu \mathrm{M}$ TTX was added to the recording saline ( $\left.1 \mathrm{mM} \mathrm{Ca}^{2+} \mathrm{HL} 3\right)$, and then evoked EJP was recorded from segment A3 muscle 1 with 1 min intervals until the response disappeared. EJP amplitudes were normalized to the amplitude before TTX addition.

\section{Dichloro-diphenyl-trichloroethane sensitivity assay}

Flies were tested for sensitivity to dichloro-diphenyl-trichloroethane (DDT) essentially as previously described (Pittendrigh et al., 1997) with some modifications. Briefly, groups of 104 -d-old flies were exposed to the insecticide in DDT-coated empty vials at $25^{\circ} \mathrm{C}$ for $12 \mathrm{~h}$. The vials were prepared by adding $10 \mu \mathrm{g}$ of DDT dissolved in acetone and drying the solvent completely. In control experiments, vials were treated with acetone alone.

\section{DSiaT $T^{H K}$ mutant}

Construct encoding DSiaT with $\mathrm{H} 406 \mathrm{~K}$ substitution $\left(\mathrm{DSiaT}^{\mathrm{HK}}\right.$ ) was generated by changing the corresponding CAT codon to AAG in the sequence encoding C-terminally HA-tagged wild-type DSiaT (DSiaT ${ }^{\text {WT }}$ ) (Koles et al., 2004). Site-specific mutagenesis was performed by PCRbased method (Panin et al., 2002). Full-length DSiaT ${ }^{W T}$ and DSiaT $T^{H K}$ were subcloned into pUAST for in vivo expression. For cell culture experiments, we generated ProtA-DSiaT ${ }^{H K}$ construct in $p M K 33$ vector applying the strategy previously used to obtain protein A-tagged wild-type DSiaT (Koles et al., 2004). 
A

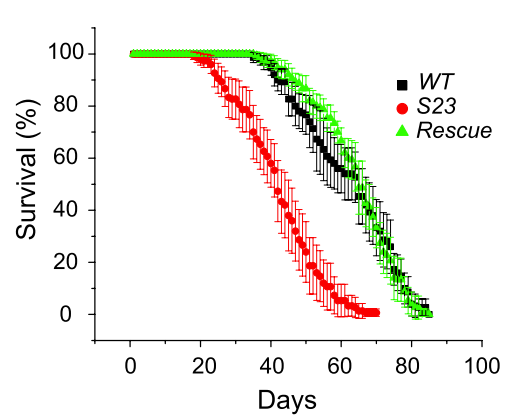

D

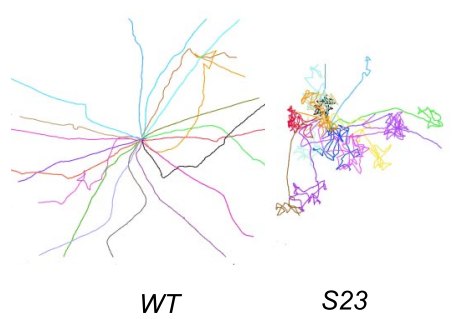

B

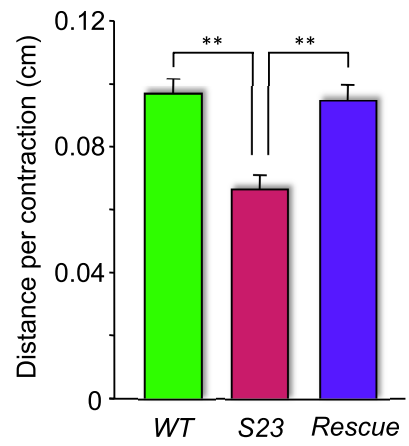

C

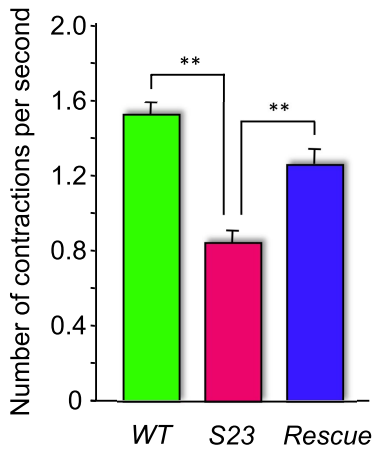

$E$

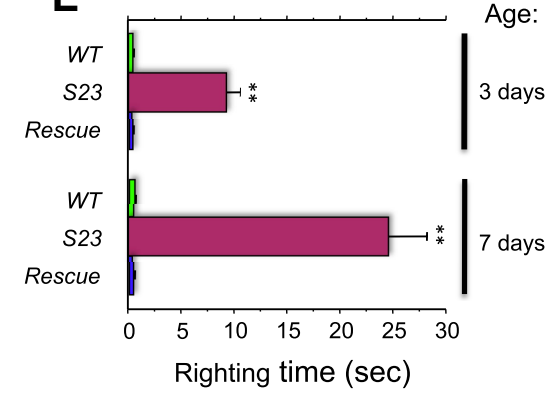

$\mathbf{F}$

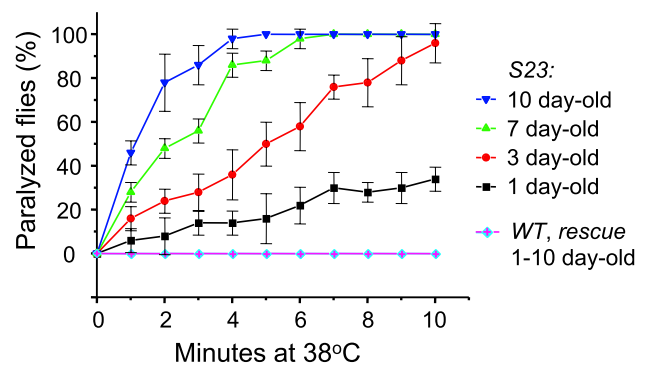

Figure 2. Behavioral phenotypes of DSiaT mutants. A, DSiaT mutants have significantly reduced longevity. For each genotype, 150 flies were assayed for group longevity; error bars represent $95 \%$ confidence intervals. $\boldsymbol{B}, \boldsymbol{C}$, Mutant larvae have crawling defects as revealed by decreased distance per muscle contraction $(\boldsymbol{B})$ and reduced speed of muscle contractions ( $\boldsymbol{C}$. Twenty larvae were assayed for each genotype. D, Mutant larvae have a crawling pattern abnormality, making many more turns. Graphs show 20 superimposed larval tracks with the initial positions overlaid in the middle. $\boldsymbol{E}$, Mutant flies have a defect in the ability of righting themselves after falling on the back. Twenty flies were assayed for each genotype. $\boldsymbol{F}$, Mutant flies have TS-paralysis phenotype. Flies were assayed at $38^{\circ} \mathrm{C}, 50$ flies per genotype. Note that the severity of locomotor defects $(\boldsymbol{E})$ and the sensitivity of mutants to elevated temperature $(\boldsymbol{F})$ significantly increased with age. In all experiments, the rescue genotype was $w^{1118} ;$ S23; P[DSiaT $\left.{ }^{+}\right]$, where P[DSiaT $\left.{ }^{+}\right]$is a transgenic insertion of wild-type DSiaT gene. Error bars represent SD in $\boldsymbol{F}$, and SEM in $\boldsymbol{B}, \boldsymbol{C}$, and $\boldsymbol{E}$. The asterisks indicate statistically significant differences ( $t$ test, $p<0.01$ ). Similar behavioral phenotypes were observed with the $L 22$ allele (supplemental Fig. S3B, available at www.jneurosci.org as supplemental material).

Protein purification and sialyltransferase assay

Cell culture expression, purification, and in vitro sialyltransferase assays of DSiaT ${ }^{\mathrm{WT}}$ and DSiaT ${ }^{\mathrm{HK}}$ were performed using previously described protocols (Koles et al., 2004).

\section{Results}

\section{Drosophila sialyltransferase mutants have decreased} longevity, locomotor abnormality, and temperature-sensitive paralysis

With only one sialyltransferase gene present in the genome (Koles et al., 2004), Drosophila represents an attractive system to explore the biological function of sialylation using reverse genetics. We generated two independent loss-of-function DSiaT alleles by introducing premature stop codons or a large deletion within the gene ORF (alleles $S 23$ and L22, respectively) via gene targeting (Maggert et al., 2008) (see Materials and Methods) (supplemental Fig. S1, available at www.jneurosci.org as supplemental material). Both mutants were homozygous viable and fertile, and did not have noticeable morphological defects. However, homozygous mutant flies were rare in balanced heterozygous stocks, which indicated their decreased viability. In addition, we found that these mutants have significantly decreased longevity (Fig. $2 A$; supplemental Fig. S2A, available at www.jneurosci.org as supplemental material) and pronounced locomotor defects, as revealed by behavioral assays in larvae and adults. Mutant larvae crawl significantly slower than wild-type and rescued mutant controls, as measured by the frequency of body muscle contractions and the distance traveled per contraction (Fig. $2 B, C$ ). In addition, mutant larvae make many more turns compared with wild-type controls when subjected to an open field crawling assay
(Fig. 2D; supplemental Fig. S2 B, available at www.jneurosci.org as supplemental material). Mutant adults have impaired coordination, and they are unable to promptly right themselves after being knocked down to the bottom of a vial by a gentle agitation (Fig. 2E), with the severity of this defect rapidly increasing with age. Since neurological mutations in Drosophila often exhibit TS paralysis (Suzuki et al., 1971), we tested the behavior of DSiaT mutants at elevated temperature and found that they become paralyzed at $38^{\circ} \mathrm{C}$ within several minutes. Paralysis is reversible as flies fully recover within $10-15$ min once temperature is shifted back to $25^{\circ} \mathrm{C}$. Similar to the locomotion phenotype, the sensitivity to elevated temperature notably increases with age (Fig. $2 \mathrm{~F}$ ), suggesting that the mutant nervous system undergoes a progressive age-dependent deterioration.

\section{DSiaT expression is restricted to a subset of CNS neurons throughout development}

To elucidate the mechanism underlying behavioral phenotypes of DSiaT mutants, we investigated the expression of DSiaT at different developmental stages. To this end, we introduced a short sequence encoding two tandem HA epitopes into the endogenous DSiaT locus using gene targeting. We found that the pattern of endogenously expressed HA-tagged DSiaT closely reflects the distribution of DSiaT mRNA detected by in situ hybridization (Koles et al., 2004, 2009). The embryonic expression of DSiaT is initiated at stage late 15 in 10-14 cells within the ventral ganglion, with the number of prominently expressing cells gradually increasing during development, until it reaches $\sim 400$ in the late third-instar larval brain (Fig. $3 A, B$ ). Dual labeling for glial 
cells with anti-Repo antibody revealed that DSiaT is not expressed in glia, whereas staining with the pan-neuronal marker Elav confirmed that all DSiaTexpressing cells are differentiated neurons (Fig. 3C-F). Although no expression was detected in dopamine and serotonin neurons labeled by dopa decarboxylase DDCGAL4 driver (data not shown) (Li et al., 2000), labeling with interneuron and motor neuron-specific drivers revealed that DSiaT is present in subsets of interneurons and motor neurons throughout the CNS (supplemental Fig. S2C, available at www.jneurosci.org as supplemental material). In addition, we found that DSiaT is expressed in the hemisegmentally repeated pattern in a previously described group of dorsomedial motor neurons in the third-instar ventral ganglion (Fig. 3G$I)$. The two motor neurons with prominent DSiaT expression were identified as MN1-Ib and MN30-Ib (Choi et al., 2004). The expression of DSiaT in MN-1b was also confirmed by labeling with RKKeveGal4 driver (Featherstone et al., 2000) (supplemental Fig. S2 D, available at www. jneurosci.org as supplemental material). In the young adult brain, DSiaT is expressed in a distinct pattern, including medulla and lobula plate regions, ocelli, the region of olfactory projection neurons, as well as some other neurons (Fig. $3 J)$. We did not detect the expression of DSiaT outside of the CNS at any developmental stage. Thus, these results suggest that DSiaT function is limited to subsets of neurons in the CNS throughout all developmental stages, which is consistent with the neurological phenotypes of DSiaT mutants.

\section{DSiaT mutants have defects in the development and physiology of neuromuscular junctions}

Many neurological mutations in Drosophila are known to impinge on the function and development of NMJs (Collins and DiAntonio, 2007). Thus, we characterized the neuroanatomy of NMJs in DSiaT mutant larvae. We focused our analyses on the dorsal abdominal muscle 1 innervated by a DSiaT-expressing motor neuron MN1-Ib. We found no targeting defects as all examined muscle 1 NMJs were innervated properly in DSiaT mutants; however, the number of branches and synaptic boutons was significantly reduced (Fig. 4A, B; supplemental Fig. S2 E, F, available at www.jneurosci.org as supplemental material), indicating a role for DSiaT function in NMJ development. The NMJ morphology defect was rescued by introducing a transgenic DSiaT genomic construct. However, the rescue was partial, which is likely attributable to the fact that the construct does not fully recapitulate the endogenous expression of DSiaT.

Since the mutant behavioral phenotypes suggested dysfunctional neural signaling, we examined neuromuscular synaptic function in DSiaT mutants using electrophysiological ap- proaches. We did not detect any significant difference in the resting membrane potential of muscle 1 between mutants and control genotypes [S23 mutants, $-60.1 \pm 0.6 \mathrm{mV}$; WT control, $-59.0 \pm 0.8 \mathrm{mV} ;$ L22 mutants, $-60.7 \pm 0.4 \mathrm{mV} ; y w$ control, $-60.1 \pm 0.3 \mathrm{mV}$ (for each genotype, errors are SEM; sample size, $N=10)$ ], which suggested that muscle functions are not affected in DSiaT mutants. Using intracellular recordings, we analyzed the EJP evoked in muscles in response to extracellular nerve stimulation. The evoked EJP amplitude was significantly decreased at mutant NMJs (Fig. 4C,D), indicating that the excitability of axonal membrane and/or the mechanism of NMJ synaptic transmission are compromised. Notably, evoked EJP was normal at muscle 6/7 NMJ innervated by MN6/7-Ib, a motor neuron without detectable DSiaT expression (supplemental Fig. $\mathrm{S} 3 A, B$, available at www.jneurosci.org as supplemental material), which suggested that DSiaT affects EJP cell-autonomously. To further investigate the mechanism underlying the defect in evoked EJP, 
A

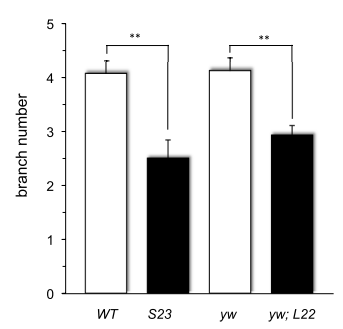

D

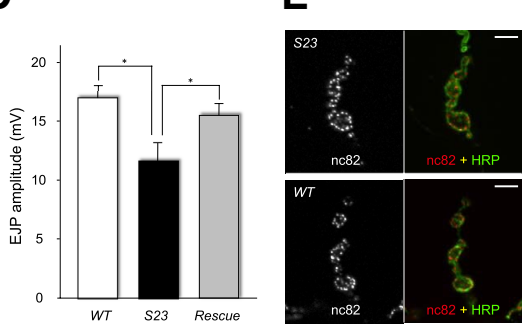

B

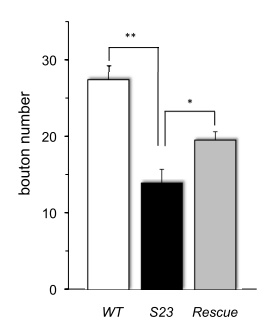

E

C

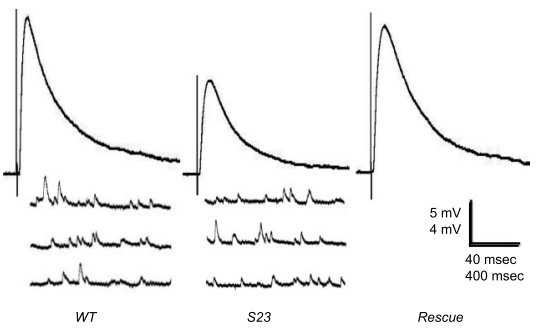

$\mathbf{F}$

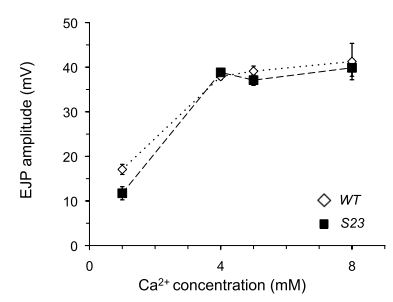

Figure 4. Morphological and electrophysiological phenotypes of DSiaT mutants. $A, B, D$ SiaT mutants have decreased number of muscle synaptic branches $(\boldsymbol{A})$ and boutons ( $\boldsymbol{B})$. $\boldsymbol{C}$, Representative traces of evoked EJPs (top) and mini-EJPs (bottom). $\boldsymbol{D}$, Evoked EJP amplitude is decreased in DSiaT mutants. Recordings were performed at $1 \mathrm{~mm} \mathrm{Ca}{ }^{2+}$ concentration. $\boldsymbol{E}$, DSiaT mutant MN1-Ib boutons have normal nc 82 staining that visualizes active zones. Anti-HRP antibody (green) was used as a presynaptic membrane marker. Images show Z-projections obtained from optical sections of boutons. Scale bar, $5 \mu \mathrm{m}$. $\boldsymbol{F}$, The defect in evoked EJP amplitude is rescued by increased $\mathrm{Ca}^{2+}$ concentration. In all panels, analyses were performed in third-instar larvae for muscle 1 NMJ in abdominal segment 3 . Error bars are SEM. The asterisks indicate statistically significant differences ( $t$ test, ${ }^{*} p<0.05$ and $\left.{ }^{* *} p<0.01\right)$. Rescue genotype was $w^{1118} ; 523 ; P\left[D S i a T^{+}\right]$. Number of larvae analyzed were as follows: $A, B: W T, 22 ; S 23,32$; Rescue, 23; yw, 29; yw; L22, 36; D, WT, 10; S23, 10; Rescue, 10; F, WT, 10; S23, 10.

we first examined mini-EJP activity as a measure of spontaneous synaptic transmission in the absence of nerve stimulation (Fig. $4 C$ ). We detected no significant difference between control and mutants in either the amplitude or frequency of mini-EJP: amplitude was $1.56 \pm 0.16$ and $1.52 \pm 0.09 \mathrm{mV}$, and frequency was $2.43 \pm 0.34$ and $2.55 \pm 0.25 \mathrm{~Hz}$ for wild-type and $S 23$ mutants, respectively (errors are SEM; $t$ test, $p>0.7$; 10 larvae were analyzed for each genotype). These results suggested that the stimulus-independent synaptic vesicle exocytosis, the amount of neurotransmitter per vesicle, and the postsynaptic sensitivity to neurotransmitter are not affected in mutants. Thus, we concluded that the decreased evoked EJP is likely associated with a defect of excitation (action potential function) at the synapse on nerve stimulation, or with abnormal synaptic functions downstream of the action potential. In general, the synaptic response depends on the number of functional active zones, the sites of neurotransmitter release, and the probability of release of synaptic vesicles at these sites (Zucker and Regehr, 2002). To test the possibility that the evoked EJP defect in DSiaT mutants might be associated with a decreased number of active zones, we labeled them with nc82 antibody [anti-Bruchpilot (Kittel et al., 2006; Wagh et al., 2006)] and estimated the density of nc 82 puncta in MN1-Ib synaptic boutons. No decrease in active zone density or the intensity of nc82 staining was found in mutants (Fig. 4E). Moreover, the density of nc 82 puncta was slightly increased in mutant boutons $\left(0.56 \pm 0.04\right.$ puncta/ $\mu \mathrm{m}^{2}$ in $S 23$ mutants vs $0.46 \pm 0.04$ puncta $/ \mu \mathrm{m}^{2}$ in wild-type control; errors are SEM; $t$ test, $p<0.0005 ; 11$ larvae were analyzed for each genotype), suggesting a possible compensatory mechanism that tends to maintain the overall strength of synaptic connection at mutant synapses with decreased NMJ size. To further shed light on synaptic processes, we measured evoked EJP amplitude in the presence of high external $\mathrm{Ca}^{2+}$ concentrations. This condition is

expected to increase the probability of synaptic vesicle fusion and transmitter release to nearly saturated level and has been shown to rescue defective EJPs with a diminished probability of release when the number of functional active zones is not effected (Wairkar et al., 2008). However, elevated $\mathrm{Ca}^{2+}$ concentration would unlikely rescue the phenotype associated with decreased number of active zones. We found that EJP amplitude reached saturation at $\geq 4 \mathrm{~mm}$ extracellular calcium concentration and that this elevated level of external $\mathrm{Ca}^{2+}$ fully rescued the EJP defect (Fig. $4 F$ ), further suggesting that the function of active zones is not affected in $D S i a T$ mutants. Moreover, this result suggests that $\mathrm{Ca}^{2+}$-triggered exocytosis is unaffected in DSiaT mutants, since increased $\mathrm{Ca}^{2+}$ concentration cannot rescue decreased neural transmission in SNARE (soluble $\mathrm{N}$-ethylmaleimide-sensitive factor attachment protein receptor) mutants (Stewart et al., 2000), as well as in several other mutants with impaired $\mathrm{Ca}^{2+}$ triggered exocytosis (Aravamudan et al., 1999; Bao et al., 2005; Long et al., 2008). To further elucidate the physiology of $\mathrm{Ca}^{2+}$ dependent excitation-secretion coupling, we analyzed short-term plasticity that is known to depend on spatiotemporal regulation of action potential-triggered $\mathrm{Ca}^{2+}$ influx (Atwood, 1967; Zucker and Regehr, 2002). We applied a paired-pulse facilitation assay that is commonly used to reveal abnormalities of synaptic transmission. In this assay, facilitation results from residual $\mathrm{Ca}^{2+}$ enhancing release probability at a closely spaced second stimulation (Katz and Miledi, 1968). The absence of facilitation could be explained by a depletion of readily releasable pool of vesicles (von Gersdorff et al., 1997). On the other hand, abnormally enhanced facilitation is indicative of a decreased probability of release at the initial pulse that results in a significant potentiation of vesicle release by accumulated intracellular $\mathrm{Ca}^{2+}$ in response to the following stimulus [as, for instance, observed in mutants with defects in synaptic vesicle release, such as bruchpilot, rotated abdomen, synaptotagmin 1, and lap (Bao et al., 2005; Kittel et al., 2006; Saraswati et al., 2007; Wairkar et al., 2008)]. We detected no difference in short-term facilitation between DSiaT mutants and wild-type controls: facilitation index was $1.48 \pm 0.07$ and $1.46 \pm 0.05$ for wild-type and DSiaT mutants, respectively (errors are SEM; $t$ test, $p>0.3 ; 19$ larvae were analyzed for each genotype) (supplemental Fig. S3C, available at www.jneurosci.org as supplemental material). This result suggested that probability of release is not affected by DSiaT. Finally, we tested the cycling of synaptic vesicles by applying high-frequency prolonged stimulation $(10 \mathrm{~Hz}, 5$ $\mathrm{min}$ ), a protocol that results in dramatic synaptic depression in mutants with defective vesicle cycling and compromised pool of synaptic vesicles (Verstreken et al., 2002, 2003; Koh et al., 2007). We did not detect any difference between wild-type control and mutants in this assay (supplemental Fig. S3D, available at www.jneurosci.org as supplemental material), indicating that the synaptic vesicle cycling is not significantly affected by DSiaT. Together, our electrophysiological results are most consistent with the conclusion that synaptic secretory mechanisms are not 
significantly affected in DSiaT mutants, while suggesting that the evoked EJP defect is a by-product of abnormal neuronal excitability because of an impaired action potential.

\section{DSiaT mutations affect the function of voltage-gated sodium channel}

To investigate the possibility that a neuronal excitability defect (i.e., compromised action potential) may underlie the physiological and behavioral phenotypes of DSiaT mutants, we have conducted additional genetic and pharmacological analyses. Action potential properties depend on the function of several families of voltagegated ion channels (Bean, 2007), with a voltage-gated $\mathrm{Na}^{+}$channel encoded by the para gene playing a major role in action potential initiation and propagation in most fly neurons (Loughney et al., 1989). Interestingly, mutations in para also result in TS paralysis (Suzuki et al., 1971), whereas a hypomorphic para mutant has a larval crawling pattern phenotype similar to that of DSiaT mutants (Fig. 2D) (Wang et al., 1997). We analyzed genetic interactions between para and DSiaT using the TS-paralysis assay and found that a copy of a temperature-sensitive conditional $(t s 1)$ or a loss-of-function ( $L K 5)$ allele of para could significantly enhance the TS-paralysis phenotype of DSiaT mutants, indicating strong synergistic interactions between these genes (Fig. 5A). No significant interaction was detected by this assay for two other TS paralytic mutants, $\operatorname{cac}^{t s 2}$ [conditional TS allele of voltage-gated $\mathrm{Ca}^{2+}$ channel $\alpha 1$ subunit (Kawasaki et al., 2002)] and tip $E^{1}$ [lossof-function allele of auxiliary subunit of voltage-gated $\mathrm{Na}^{+}$channel (Feng et al., 1995)] (supplemental Fig. S4, available at www. jneurosci.org as supplemental material). These results further supported the specificity of genetic interactions between para and DSiaT, suggesting that the function of Para is affected in DSiaT mutants. To examine this effect more directly, we used a pharmacological strategy based on the specific inhibition of Para by TTX, a neurotoxin that blocks the channel by binding its pore loops with high affinity (Catterall, 2000). We hypothesized that if Para properties are changed in DSiaT mutants, it may result in an altered TTX-mediated inhibition of Para. Indeed, we found that the kinetics of inhibition measured by the gradual disappearance of the stimulus-evoked EJP in muscle 1 after TTX addition was significantly slower in DSiaT mutants than in wild-type controls (Fig. $5 B, C$ ). Yet another evidence suggesting that Para function is affected in DSiaT mutants came from the decreased sensitivity of the mutants to DDT (Fig. 5D; supplemental Fig. S3E, available at www.jneurosci.org as supplemental material), an insecticide that specifically interacts with voltage-gated sodium channels and alters their gating (Davies et al., 2007). The DDT sensitivity phenotype was rescued by the DSiaT transgene (the rescue was incomplete, probably because the transgene did not fully restore the endogenous level of DSiaT activity). Together, these results indicate that DSiaT regulates the functional properties of Para, strongly suggesting that decreased neural transmission in DSiaT mutant neurons is associated with altered neuronal excitability because of an action potential defect.

\section{Sialyltransferase activity is required for DSiaT function in vivo}

We tested whether the sialyltransferase activity of the DSiaT protein is important for its function in vivo. To this end, we embarked on generating an enzymatically inactive form of DSiaT that would still be correctly folded, retaining an overall normal structure. A conserved histidine residue within the VS sialylmotif was proposed to be involved in the catalytic activity of mammalian sialyltransferases (Kitazume-Kawaguchi et al., 2001). Mutations of this residue inactivated mammalian sialyltransferases, while preserving their folding and substrate binding (KitazumeKawaguchi et al., 2001; Jeanneau et al., 2004). Thus, we generated an expression construct encoding $\mathrm{DSiaT}^{\mathrm{HK}}$, a point mutant with the conserved His ${ }^{406}$ in the VS motif changed to Lys (supplemental Fig. S5, available at www.jneurosci.org as supplemental material). This mutant was expressed as a protein A-tagged secreted construct in Drosophila S2 cells, purified, and assayed for enzymatic activity in vitro. We found that the mutant protein has no detectable sialyltransferase activity (Fig. 6A). At the same time, when the full-length $\mathrm{DSiaT}{ }^{\mathrm{HK}}$ protein was expressed in vivo using UAS-GAL4 system, the level of expression and subcellular localization of $\mathrm{DSiaT}^{\mathrm{HK}}$ were indistinguishable from those of the matching wild-type control, DSiaT ${ }^{\text {WT }}$ (Fig. $6 \mathrm{~B}$ ), suggesting that the $\mathrm{H} 406 \mathrm{~K}$ mutation indeed does not affect the folding and proper localization of the sialyltransferase protein within the cell. 
A

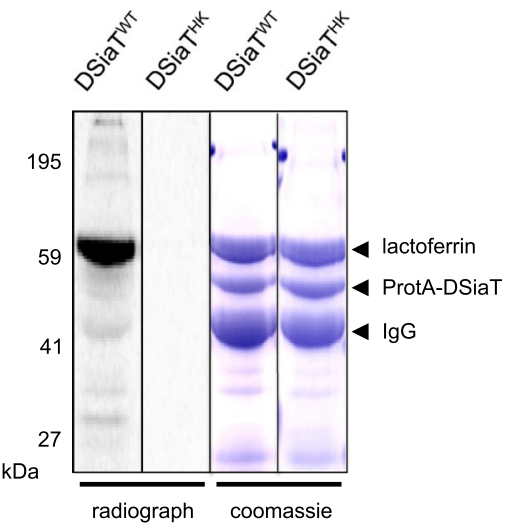

B

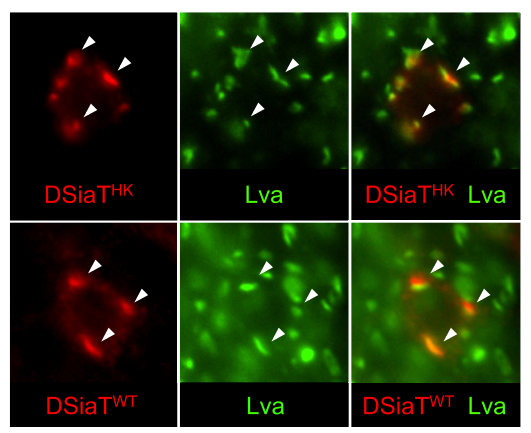

C

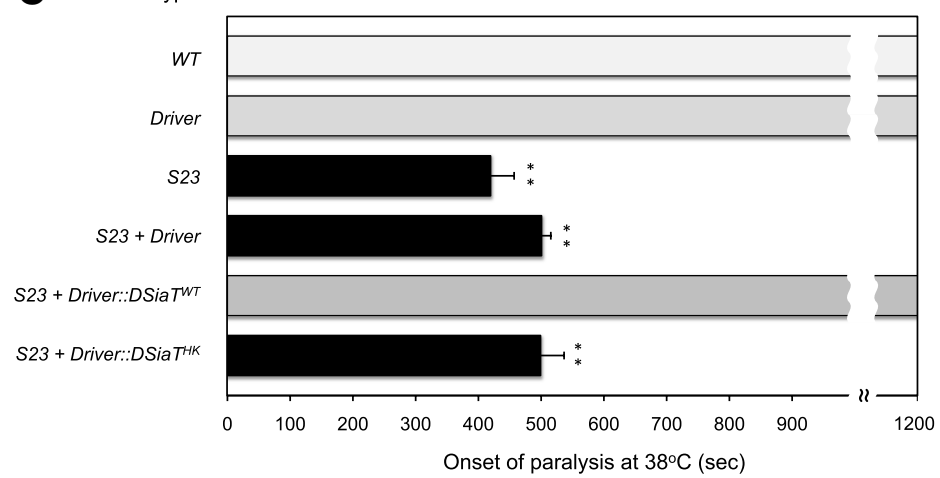

D Genotype:

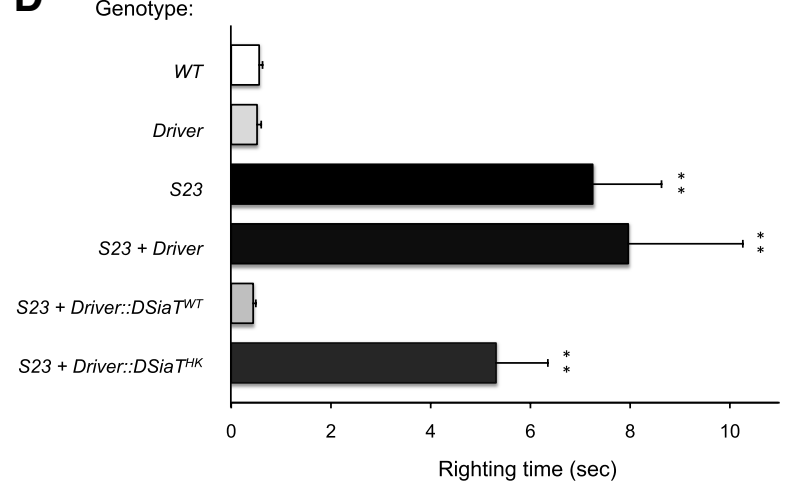

Figure 6. Sialyltransferase activity is required for in vivo DSiaT functioning. A, DSiaT ${ }^{\mathrm{HK}}$ mutant protein has no detectable activity in vitro. DSiaT ${ }^{\mathrm{HK}}$ and DSiaT ${ }^{\mathrm{WT}}$ expressed in Drosophila S2 cells as protein A-fusion constructs were assayed for sialyltransferase activity using $C M P-\left[{ }^{14} \mathrm{C}\right]$ Sia as a donor and lactoferrin as an acceptor. The left two lanes are the autoradiograph of products of the assays separated on SDS gel; the right two lanes show the Coomassie staining of the same gel as a control for protein amount. IgG, Ig leached from beads used in purification (Koles et al., 2004). B, DSiat ${ }^{\mathrm{HK}}$ is expressed and localized normally in brain neurons. DSiaT ${ }^{\mathrm{HK}}$ and DSiaT ${ }^{\mathrm{WT}}$ were expressed in vivo as C-terminally HA-tagged full-length constructs using C155-Gal 4 driver. There is no difference in the level of expression or subcellular localization of these proteins, as revealed by double staining with a Golgi marker, Lva (green) (Sisson et al., 2000). C, The transgenic expression of DSiaT wild-type construct with a neuronal driver fully rescues the TS-paralysis phenotype of DSiaT mutants; however, the expression of enzymatically inactive DSiaT protein, DSiaT ${ }^{\mathrm{HK}}$, is unable to rescue the mutant phenotype. $D$, Sialyltransferase activity is required for rescuing locomotor defect of DSiaT mutants. Whereas transgenic DSiaT ${ }^{\mathrm{WT}}$ fully rescues locomotor abnormalities, DSiaT ${ }^{\mathrm{HK}}$ is unable to rescue the mutant phenotype. Genotypes are as follows: WT, Wild-type control (w ${ }^{1118}$ Canton S); Driver, C155-GAL4/+; S23+Driver::DSiaT ${ }^{W T}, \quad\left(155-G A L 4 /+; S 23 / S 23 ; U A S-D S i a T^{W T} /+\right.$; S23+Driver::DsiaT ${ }^{H K},\left(155-G A L 4 /+; S 23 / S 23 ; U A S-D S i a T^{H K} /+\right.$. Twenty 5-d-old flies were assayed for each genotype. Error bars are SEM. The asterisks indicate the results with significant differences from wild-type phenotypes ( $t$ test, ${ }^{* *} p<0.01$ ) (but these results are not significantly different from each other).

Yet the expression of DSiaT ${ }^{\mathrm{HK}}$ was unable to rescue the TS paralysis and locomotor abnormalities of DSiaT mutants (Fig. 6C,D). Thus, these results demonstrated that the sialyltransferase activity of DSiaT is essential for its biological function.

\section{Discussion}

Our results revealed that Drosophila sialyltransferase functions specifically in the CNS throughout development, modulating excitability of individual neurons, influencing NMJ development, and affecting behaviors and longevity at the organismal level. This is the first demonstration, to our knowledge, of a specific biological role of an ST6Gal sialyltransferase in the nervous system. Mammals have two ST6Gal enzymes, with one of them, ST6Gal I, being implicated in immune system function and cancer (Hennet et al., 1998; Hedlund et al., 2008). No mutants have been yet characterized for the second enzyme, ST6Gal II, whose function remains unknown. Whereas ST6Gal I is expressed ubiquitously, ST6Gal II has a tissue-specific expression, with elevated level being detected in the fetal and adult brain (Takashima et al., 2002; Krzewinski-Recchi et al., 2003). Based on its structural and enzymatic properties (Takashima et al., 2002; Krzewinski-Recchi et al., 2003; Rohfritsch et al., 2006), ST6Gal II represents the closest mammalian counterpart of DSiaT (Fig. 1) (Koles et al.,
2004). Thus, our results suggest an intriguing possibility that ST6Gal II, similar to its Drosophila homolog, may be involved in the regulation of nervous system function, which will require additional investigation.

DSiaT has a substantial impact on CNS function at the organismal level. Although DSiaT activity is required for properly coordinated locomotion and longevity under normal conditions, its effect on neural functions is compounded at elevated temperature. Together, these results implicate DSiaT-mediated sialylation in essential neuronal network regulation. Moreover, the severity of behavioral phenotypes in DSiaT mutants rapidly increases with age, which suggests a progressive deterioration of neural functions and potential neurodegeneration. This is consistent with significantly decreased life span of DSiaT mutants, and with the fact that the mutants exhibiting TS paralysis and defects in membrane excitability commonly have neurodegeneration (Palladino et al., 2002; Fergestad et al., 2006, 2008). Additional studies will reveal the cellular mechanisms underlying the age-dependent exacerbation of behavioral phenotypes in DSiaT mutants.

Our analysis of larval NMJ morphology revealed a decreased number of synaptic boutons and branches in DSiaT mutants, which suggests that DSiaT activity promotes the growth of syn- 
aptic connections. It is interesting that, despite the decreased size of the neuromuscular synapses, the synaptic activity appears to be properly maintained in DSiaT mutants at the whole-muscle level, as suggested by increased density of active zones, normal miniEJP, unchanged pared-pulsed facilitation, and proper synaptic vesicle dynamics, as well as by the rescue of evoked EJP phenotype by increased $\mathrm{Ca}^{2+}$ concentration. This conclusion is consistent with the fact that transgenic DSiaT construct can fully rescue the EJP defect, albeit it does not completely restore NMJ morphology (likely because its expression does not fully recapitulate the endogenous DSiaT expression during NMJ development), which indicates that the decreased EJP and abnormal NMJ are two separable phenotypes. The effect of DSiaT on synaptic morphology may be explained by a decrease in neural excitability during the growth and development of larval NMJ synapses. Neural excitability was shown to positively regulate the postembryonic development of motor terminals, whereas reduced impulse activity results in smaller NMJs (Budnik et al., 1990; Sigrist et al., 2003; Zhong and Wu, 2004; Guan et al., 2005). Moreover, genetic manipulations with para activity revealed that the increased level of para results in NMJ synapse overgrowth, while reducing para activity (e.g., using nap mutations), has an opposite effect, leading to a decreased number of branches and fully suppressing the NMJ overgrowth induced by elevated temperature or by the mutations resulting in membrane hyperexcitability, such as eag and Sh (Budnik et al., 1990; Zhong and Wu, 2004). These data are consistent with our results indicating that DSiaT affects Para function.

We demonstrated that sialyltransferase activity is required for DSiaT in vivo function, which indicates that DSiaT mutant phenotypes result from a deficiency of sialylation. To our knowledge, this is the first evidence that sialylation has an important biological function in protostomes. Previous research on sialylation in insect cells has been controversial, with some experiments suggesting the presence of sialylated structures or biochemical potential for sialylation, and others unable to detect sialic acids (Marchal et al., 2001; Angata and Varki, 2002; Koles et al., 2009). The absence of reliable evidence of sialylation or its function in protostomes has led to the long-standing paradigm that sialic acid biosynthesis is a late evolutionary invention of deuterostomes (vertebrates, ascidians, and echinoderms), whereas protostomes, including Drosophila, are beings devoid of sialic acids or any important role of sialylation (Angata and Varki, 2002). Although more recently sialylated structures have been unambiguously detected in Drosophila (Aoki et al., 2007; Koles et al., 2007), their biological role remained elusive. Our results shed light on the function of sialylation in Drosophila and suggest that sialylation is tightly regulated by spatially and temporally restricted expression of DSiaT throughout development. These results also indicate that sialylated glycans may be confined in Drosophila to a subset of CNS neurons.

Using behavioral, genetic, electrophysiological, and pharmacological approaches, we present four different lines of evidence that DSiaT activity impinges on the function of Para, a voltagegated sodium channel. A possible scenario involves a direct mechanism of sialylation of putative $\mathrm{N}$-linked glycans located on Para pore loops (Loughney et al., 1989). The potential sialylation of pore loops may influence Para interactions with TTX and DDT, or/and it may modify channel gating, thus affecting the efficacy of their action. This possibility is consistent with the fact that single amino acid substitutions within the pore loops can confer resistance to TTX and DDT (Pittendrigh et al., 1997; Catterall, 2000). In vertebrates, voltage-gated $\mathrm{Na}^{+}$channels are known to be heavily sialylated (Miller et al., 1983; James and
Agnew, 1987; Schmidt and Catterall, 1987), with sialic acids being responsible for changes in gating properties of the channels in vitro (Recio-Pinto et al., 1990) and in cell culture in an isoformspecific manner (Bennett, 2002). In rat models, the channel glycosylation was found to be developmentally regulated, and treatments with glycosidases that remove sialylated glycans suggested that the membrane sialylation modulates voltage-gated $\mathrm{Na}^{+}$channels in dorsal root ganglion neurons (Tyrrell et al., 2001) and in the hippocampus, while influencing network excitability and seizure threshold in kindling epilepsy models (Isaev et al., 2007). Our experiments demonstrated that sialylation plays an important role in Drosophila CNS, regulating excitability of neuronal membranes and modulating the function of a voltagegated $\mathrm{Na}^{+}$channel. These findings not only reveal the evolutionary conservation of the biological function of sialylation between Drosophila and mammals but also present the first in vivo evidence that $\mathrm{N}$-linked sialylation plays a specific role in the control of neuronal transmission, while demonstrating the significance of such a role at the organismal level. Importantly, our results suggest a novel function of sialylation in the regulation of voltagegated $\mathrm{Na}^{+}$channel since DSiaT-generated structures are predicted to be monosialylated biantennary N-glycans (Koles et al., 2004, 2007; Aoki et al., 2007), as opposed to the heavily sialylated carbohydrates modifications present on vertebrate voltage-gated $\mathrm{Na}^{+}$channels (Miller et al., 1983; Roberts and Barchi, 1987). So far, the effect of sialylation on the function of voltage-gated $\mathrm{Na}^{+}$ channels in neurons has been attributed to a large negative charge caused by a significant number of sialic acid residues attached to the channel [estimated as $>100$ residues per channel (Miller et al., 1983; Bennett, 2002)], including polymers of sialic acid (James and Agnew, 1987; Zuber et al., 1992). Our data, however, imply that sialylation can have a more specific effect on ion channel function, not necessarily associated with a significant charge accumulated in the vicinity of channel pore, and suggest that sialylated $\mathrm{N}$-glycans may have a novel role in regulating neural excitability. Such a specific function of sialylation in modulating the voltage-gated sodium channel is consistent with the fact that Endo- $\mathrm{N}$ treatment that specifically removes polysialic acids has no effect on evoked synaptic transmission and intracellularly recorded action potentials in rat hippocampus (Muller et al., 1996). This function may be also involved in the regulation of cardiac sodium channel, which is significantly less sialylated than its neuronal counterparts and whose properties appear to be affected by certain "functional" sialic acids (Stocker and Bennett, 2006) rather than by a total surface charge accumulated because of sialylation (Ufret-Vincenty et al., 2001; Bennett, 2002; Fozzard and Kyle, 2002). Molecular mechanisms underlying the role of sialylated N-glycans in the function of Para, including direct analysis of its potential sialylation, will require additional investigation, while taking into account that a large number of Para variants can be produced by alternative splicing (Olson et al., 2008) and RNA editing (Palladino et al., 2000), and that these variants may be differently affected by DSiaT. Together, our results suggest that Drosophila can be a useful and experimentally amenable model for elucidating the function of sialylation in the nervous system.

\section{References}

Abramoff MD, Magelhaes PJ, Ram SJ (2004) Image processing with ImageJ. Biophotonics Int 11:36042.

Alfonso TB, Jones BW (2002) gcm2 promotes glial cell differentiation and is required with glial cells missing for macrophage development in Drosophila. Dev Biol 248:369-383.

Angata K, Long JM, Bukalo O, Lee W, Dityatev A, Wynshaw-Boris A, Schachner M, Fukuda M, Marth JD (2004) Sialyltransferase ST8Sia-II 
assembles a subset of polysialic acid that directs hippocampal axonal targeting and promotes fear behavior. J Biol Chem 279:32603-32613.

Angata T, Varki A (2002) Chemical diversity in the sialic acids and related alpha-keto acids: an evolutionary perspective. Chem Rev 102:439-469.

Aoki K, Perlman M, Lim JM, Cantu R, Wells L, Tiemeyer M (2007) Dynamic developmental elaboration of $\mathrm{N}$-linked glycan complexity in the Drosophila melanogaster embryo. J Biol Chem 282:9127-9142.

Aravamudan B, Fergestad T, Davis WS, Rodesch CK, Broadie K (1999) Drosophila UNC-13 is essential for synaptic transmission. Nat Neurosci 2:965-971.

Atwood HL (1967) Variation in physiological properties of crustacean motor synapses. Nature 215:57-58.

Bao H, Daniels RW, MacLeod GT, Charlton MP, Atwood HL, Zhang B (2005) AP180 maintains the distribution of synaptic and vesicle proteins in the nerve terminal and indirectly regulates the efficacy of $\mathrm{Ca}^{2+}$. triggered exocytosis. J Neurophysiol 94:1888-1903.

Bean BP (2007) The action potential in mammalian central neurons. Nat Rev Neurosci 8:451-465.

Bennett ES (2002) Isoform-specific effects of sialic acid on voltage-dependent $\mathrm{Na}^{+}$channel gating: functional sialic acids are localized to the S5-S6 loop of domain I. J Physiol 538:675-690.

Brooks IM, Felling R, Kawasaki F, Ordway RW (2003) Genetic analysis of a synaptic calcium channel in Drosophila: intragenic modifiers of a temperaturesensitive paralytic mutant of cacophony. Genetics 164:163-171.

Budnik V, Zhong Y, Wu CF (1990) Morphological plasticity of motor axons in Drosophila mutants with altered excitability. J Neurosci 10:3754-3768.

Budnik V, Koh YH, Guan B, Hartmann B, Hough C, Woods D, Gorczyca M (1996) Regulation of synapse structure and function by the Drosophila tumor suppressor gene dlg. Neuron 17:627-640.

Catterall WA (2000) From ionic currents to molecular mechanisms: the structure and function of voltage-gated sodium channels. Neuron 26:13-25.

Choi JC, Park D, Griffith LC (2004) Electrophysiological and morphological characterization of identified motor neurons in the Drosophila third instar larva central nervous system. J Neurophysiol 91:2353-2365.

Collins CA, DiAntonio A (2007) Synaptic development: insights from Drosophila. Curr Opin Neurobiol 17:35-42.

Daniels RW, Gelfand MV, Collins CA, DiAntonio A (2008) Visualizing glutamatergic cell bodies and synapses in Drosophila larval and adult CNS. J Comp Neurol 508:131-152.

Davies TG, Field LM, Usherwood PN, Williamson MS (2007) DDT, pyrethrins, pyrethroids and insect sodium channels. IUBMB Life 59:151-162.

Dubnau J, Grady L, Kitamoto T, Tully T (2001) Disruption of neurotransmission in Drosophila mushroom body blocks retrieval but not acquisition of memory. Nature 411:476-480.

Eckhardt M, Bukalo O, Chazal G, Wang L, Goridis C, Schachner M, GerardySchahn R, Cremer H, Dityatev A (2000) Mice deficient in the polysialyltransferase ST8SiaIV/PST-1 allow discrimination of the roles of neural cell adhesion molecule protein and polysialic acid in neural development and synaptic plasticity. J Neurosci 20:5234-5244.

Featherstone DE, Rushton EM, Hilderbrand-Chae M, Phillips AM, Jackson FR, Broadie K (2000) Presynaptic glutamic acid decarboxylase is required for induction of the postsynaptic receptor field at a glutamatergic synapse. Neuron 27:71-84.

Feng G, Deák P, Chopra M, Hall LM (1995) Cloning and functional analysis of TipE, a novel membrane protein that enhances Drosophila para sodium channel function. Cell 82:1001-1011.

Fergestad T, Ganetzky B, Palladino MJ (2006) Neuropathology in Drosophila membrane excitability mutants. Genetics 172:1031-1042.

Fergestad T, Olson L, Patel KP, Miller R, Palladino MJ, Ganetzky B (2008) Neuropathology in Drosophila mutants with increased seizure susceptibility. Genetics 178:947-956.

Fozzard HA, Kyle JW (2002) Do defects in ion channel glycosylation set the stage for lethal cardiac arrhythmias? Sci STKE 2002:pe19.

Ganetzky B (1984) Genetic studies of membrane excitability in Drosophila: lethal interaction between two temperature-sensitive paralytic mutations. Genetics 108:897-911.

Gong WJ, Golic KG (2003) Ends-out, or replacement, gene targeting in Drosophila. Proc Natl Acad Sci U S A 100:2556-2561.

Gong WJ, Golic KG (2004) Genomic deletions of the Drosophila melanogaster Hsp70 genes. Genetics 168:1467-1476.

Guan Z, Saraswati S, Adolfsen B, Littleton JT (2005) Genome-wide tran- scriptional changes associated with enhanced activity in the Drosophila nervous system. Neuron 48:91-107.

Haines N, Stewart BA (2007) Functional roles for $\beta 1,4-N$-acetlygalactosaminyltransferase-A in Drosophila larval neurons and muscles. Genetics 175:671-679.

Harduin-Lepers A, Vallejo-Ruiz V, Krzewinski-Recchi MA, Samyn-Petit B, Julien S, Delannoy P (2001) The human sialyltransferase family. Biochimie 83:727-737.

Hedlund M, Ng E, Varki A, Varki NM (2008) $\alpha 2$-6-Linked sialic acids on $\mathrm{N}$-glycans modulate carcinoma differentiation in vivo. Cancer Res 68:388-394.

Hennet T, Chui D, Paulson JC, Marth JD (1998) Immune regulation by the ST6Gal sialyltransferase. Proc Natl Acad Sci U S A 95:4504-4509.

Hildebrandt H, Mühlenhoff M, Weinhold B, Gerardy-Schahn R (2007) Dissecting polysialic acid and NCAM functions in brain development. J Neurochem 103 [Suppl 1]:S56-S64.

Isaev D, Isaeva E, Shatskih T, Zhao Q, Smits NC, Shworak NW, Khazipov R, Holmes GL (2007) Role of extracellular sialic acid in regulation of neuronal and network excitability in the rat hippocampus. J Neurosci 27:11587-11594.

James WM, Agnew WS (1987) Multiple oligosaccharide chains in the voltagesensitive Na channel from electrophorus electricus: evidence for $\alpha$-2,8-linked polysialic acid. Biochem Biophys Res Commun 148:817-826.

Jan LY, Jan YN (1982) Antibodies to horseradish peroxidase as specific neuronal markers in Drosophila and in grasshopper embryos. Proc Natl Acad Sci U S A 79:2700-2704.

Jeanneau C, Chazalet V, Augé C, Soumpasis DM, Harduin-Lepers A, Delannoy P, Imberty A, Breton C (2004) Structure-function analysis of the human sialyltransferase ST3Gal I: role of N-glycosylation and a novel conserved sialylmotif. J Biol Chem 279:13461-13468.

Katz B, Miledi R (1968) The role of calcium in neuromuscular facilitation. J Physiol 195:481-492.

Kawasaki F, Collins SC, Ordway RW (2002) Synaptic calcium-channel function in Drosophila: analysis and transformation rescue of temperaturesensitive paralytic and lethal mutations of cacophony. J Neurosci 22:5856-5864.

Kim K, Lawrence SM, Park J, Pitts L, Vann WF, Betenbaugh MJ, Palter KB (2002) Expression of a functional Drosophila melanogaster $\mathrm{N}$-acetylneuraminic acid (Neu5Ac) phosphate synthase gene: evidence for endogenous sialic acid biosynthetic ability in insects. Glycobiology 12:73-83.

Kitazume-Kawaguchi S, Kabata S, Arita M (2001) Differential biosynthesis of polysialic or disialic acid structure by ST8Sia II and ST8Sia IV. J Biol Chem 276:15696-15703.

Kittel RJ, Wichmann C, Rasse TM, Fouquet W, Schmidt M, Schmid A, Wagh DA, Pawlu C, Kellner RR, Willig KI, Hell SW, Buchner E, Heckmann M, Sigrist SJ (2006) Bruchpilot promotes active zone assembly, $\mathrm{Ca}^{2+}$ channel clustering, and vesicle release. Science 312:1051-1054.

Koh TW, Korolchuk VI, Wairkar YP, Jiao W, Evergren E, Pan H, Zhou Y, Venken KJ, Shupliakov O, Robinson IM, O'Kane CJ, Bellen HJ (2007) Eps15 and Dap160 control synaptic vesicle membrane retrieval and synapse development. J Cell Biol 178:309-322.

Koles K, Irvine KD, Panin VM (2004) Functional characterization of Drosophila sialyltransferase. J Biol Chem 279:4346-4357.

Koles K, Lim JM, Aoki K, Porterfield M, Tiemeyer M, Wells L, Panin V (2007) Identification of N-glycosylated proteins from the central nervous system of Drosophila melanogaster. Glycobiology 17:1388-1403.

Koles K, Repnikova E, Pavlova G, Korochkin LI, Panin VM (2009) Sialylation in protostomes: a perspective from Drosophila genetics and biochemistry. Glycoconj J 26:313-324.

Krzewinski-Recchi MA, Julien S, Juliant S, Teintenier-Lelièvre M, Samyn-Petit B, Montiel MD, Mir AM, Cerutti M, Harduin-Lepers A, Delannoy P (2003) Identification and functional expression of a second human $\beta$-galactoside $\alpha$ 2,6-sialyltransferase, ST6Gal II. Eur J Biochem 270:950-961.

Larkin MA, Blackshields G, Brown NP, Chenna R, McGettigan PA, McWilliam H, Valentin F, Wallace IM, Wilm A, Lopez R, Thompson JD, Gibson TJ, Higgins DG (2007) Clustal W and Clustal X version 2.0. Bioinformatics 23:2947-2948

Li H, Chaney S, Roberts IJ, Forte M, Hirsh J (2000) Ectopic G-protein expression in dopamine and serotonin neurons blocks cocaine sensitization in Drosophila melanogaster. Curr Biol 10:211-214.

Long AA, Kim E, Leung HT, Woodruff E 3rd, An L, Doerge RW, Pak WL, Broadie K (2008) Presynaptic calcium channel localization and calcium- 
dependent synaptic vesicle exocytosis regulated by the Fuseless protein. J Neurosci 28:3668-3682.

Loughney K, Kreber R, Ganetzky B (1989) Molecular analysis of the para locus, a sodium channel gene in Drosophila. Cell 58:1143-1154.

Lyalin D, Koles K, Roosendaal SD, Repnikova E, Van Wechel L, Panin VM (2006) The twisted gene encodes Drosophila protein O-mannosyltransferase 2 and genetically interacts with the rotated abdomen gene encoding Drosophila protein $\mathrm{O}$-mannosyltransferase 1. Genetics 172:343-353.

Maggert KA, Gong WJ, Golic KG (2008) Methods for homologous recombination in Drosophila. Methods Mol Biol 420:155-174.

Marchal I, Jarvis DL, Cacan R, Verbert A (2001) Glycoproteins from insect cells: sialylated or not? Biol Chem 382:151-159.

Martin LT, Marth JD, Varki A, Varki NM (2002) Genetically altered mice with different sialyltransferase deficiencies show tissue-specific alterations in sialylation and sialic acid 9-O-acetylation. J Biol Chem 277: 32930-32938.

Miller JA, Agnew WS, Levinson SR (1983) Principal glycopeptide of the tetrodotoxin/saxitoxin binding protein from Electrophorus electricus: isolation and partial chemical and physical characterization. Biochemistry 22:462-470.

Moody AM, Chui D, Reche PA, Priatel JJ, Marth JD, Reinherz EL (2001) Developmentally regulated glycosylation of the CD8alphabeta coreceptor stalk modulates ligand binding. Cell 107:501-512.

Mühlenhoff M, Oltmann-Norden I, Weinhold B, Hildebrandt H, GerardySchahn R (2009) Brain development needs sugar: the role of polysialic acid in controlling NCAM functions. Biol Chem 390:567-574.

Muller D, Wang C, Skibo G, Toni N, Cremer H, Calaora V, Rougon G, Kiss JZ (1996) PSA-NCAM is required for activity-induced synaptic plasticity. Neuron 17:413-422.

Niman HL, Houghten RA, Walker LE, Reisfeld RA, Wilson IA, Hogle JM, Lerner RA (1983) Generation of protein-reactive antibodies by short peptides is an event of high frequency: implications for the structural basis of immune recognition. Proc Natl Acad Sci U S A 80:4949-4953.

Olson RO, Liu Z, Nomura Y, Song W, Dong K (2008) Molecular and functional characterization of voltage-gated sodium channel variants from Drosophila melanogaster. Insect Biochem Mol Biol 38:604-610.

Palladino MJ, Keegan LP, O'Connell MA, Reenan RA (2000) A-to-I premRNA editing in Drosophila is primarily involved in adult nervous system function and integrity. Cell 102:437-449.

Palladino MJ, Hadley TJ, Ganetzky B (2002) Temperature-sensitive paralytic mutants are enriched for those causing neurodegeneration in Drosophila. Genetics 161:1197-1208.

Panin VM, Shao L, Lei L, Moloney DJ, Irvine KD, Haltiwanger RS (2002) Notch ligands are substrates for protein $O$-fucosyltransferase- 1 and Fringe. J Biol Chem 277:29945-29952.

Pittendrigh B, Reenan R, ffrench-Constant RH, Ganetzky B (1997) Point mutations in the Drosophila sodium channel gene para associated with resistance to DDT and pyrethroid insecticides. Mol Gen Genet 256:602-610.

Recio-Pinto E, Thornhill WB, Duch DS, Levinson SR, Urban BW (1990) Neuraminidase treatment modifies the function of electroplax sodium channels in planar lipid bilayers. Neuron 5:675-684.

Roberts RH, Barchi RL (1987) The voltage-sensitive sodium channel from rabbit skeletal muscle. Chemical characterization of subunits. J Biol Chem 262:2298-2303.

Rohfritsch PF, Joosten JA, Krzewinski-Recchi MA, Harduin-Lepers A, Laporte B, Juliant S, Cerutti M, Delannoy P, Vliegenthart JF, Kamerling JP (2006) Probing the substrate specificity of four different sialyltransferases using synthetic $\beta$-D-Galp- $(1 \rightarrow 4)-\beta$-D-GlcpNAc- $(1 \rightarrow 2)-\alpha$-D-

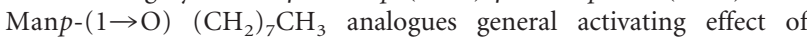
replacing $\mathrm{N}$-acetylglucosamine by $\mathrm{N}$-propionylglucosamine. Biochim Biophys Acta 1760:685-692.

Rong YS, Golic KG (2000) Gene targeting by homologous recombination in Drosophila. Science 288:2013-2018.

Rutishauser U (2008) Polysialic acid in the plasticity of the developing and adult vertebrate nervous system. Nat Rev Neurosci 9:26-35.

Saraswati S, Adolfsen B, Littleton JT (2007) Characterization of the role of the Synaptotagmin family as calcium sensors in facilitation and asynchronous neurotransmitter release. Proc Natl Acad Sci U S A 104:14122-14127.

Schmidt JW, Catterall WA (1987) Palmitylation, sulfation, and glycosylation of the alpha subunit of the sodium channel. Role of post-translational modifications in channel assembly. J Biol Chem 262:13713-13723.

Sigrist SJ, Reiff DF, Thiel PR, Steinert JR, Schuster CM (2003) Experience- dependent strengthening of Drosophila neuromuscular junctions. J Neurosci 23:6546-6556.

Sisson JC, Field C, Ventura R, Royou A, Sullivan W (2000) Lava lamp, a novel peripheral golgi protein, is required for Drosophila melanogaster cellularization. J Cell Biol 151:905-918.

Song W, Ranjan R, Dawson-Scully K, Bronk P, Marin L, Seroude L, Lin YJ, Nie Z, Atwood HL, Benzer S, Zinsmaier KE (2002) Presynaptic regulation of neurotransmission in Drosophila by the G protein-coupled receptor methuselah. Neuron 36:105-119.

Stewart BA, McLean JR (2004) Population density regulates Drosophila synaptic morphology in a Fasciclin-II-dependent manner. J Neurobiol 61:392-399.

Stewart BA, Atwood HL, Renger JJ, Wang J, Wu CF (1994) Improved stability of Drosophila larval neuromuscular preparations in haemolymphlike physiological solutions. J Comp Physiol A Neuroethol Sens Neural Behav Physiol 175:179-191.

Stewart BA, Mohtashami M, Trimble WS, Boulianne GL (2000) SNARE proteins contribute to calcium cooperativity of synaptic transmission. Proc Natl Acad Sci U S A 97:13955-13960.

Stocker PJ, Bennett ES (2006) Differential sialylation modulates voltagegated $\mathrm{Na}^{+}$channel gating throughout the developing myocardium. J Gen Physiol 127:253-265.

Suzuki DT, Grigliatti T, Williamson R (1971) Temperature-sensitive mutations in Drosophila melanogaster. VII. A mutation (para-ts) causing reversible adult paralysis. Proc Natl Acad Sci U S A 68:890-893.

Takashima S, Tsuji S, Tsujimoto M (2002) Characterization of the second type of human $\beta$-galactoside $\alpha 2,6$-sialyltransferase (ST6Gal II), which sialylates $\mathrm{Gal} \beta 1,4 \mathrm{GlcNAc}$ structures on oligosaccharides preferentially. J Biol Chem 277:45719-45728.

Tyrrell L, Renganathan M, Dib-Hajj SD, Waxman SG (2001) Glycosylation alters steady-state inactivation of sodium channel $\mathrm{Nav1} .9 / \mathrm{NaN}$ in dorsal root ganglion neurons and is developmentally regulated. J Neurosci 21:9629-9637.

Ufret-Vincenty CA, Baro DJ, Lederer WJ, Rockman HA, Quinones LE, Santana LF (2001) Role of sodium channel deglycosylation in the genesis of cardiac arrhythmias in heart failure. J Biol Chem 276:28197-28203.

Varki A (2007) Glycan-based interactions involving vertebrate sialic-acidrecognizing proteins. Nature 446:1023-1029.

Varki A (2008) Sialic acids in human health and disease. Trends Mol Med 14:351-360.

Verstreken P, Kjaerulff O, Lloyd TE, Atkinson R, Zhou Y, Meinertzhagen IA, Bellen HJ (2002) Endophilin mutations block clathrin-mediated endocytosis but not neurotransmitter release. Cell 109:101-112.

Verstreken P, Koh TW, Schulze KL, Zhai RG, Hiesinger PR, Zhou Y, Mehta SQ, Cao Y, Roos J, Bellen HJ (2003) Synaptojanin is recruited by endophilin to promote synaptic vesicle uncoating. Neuron 40:733-748.

Viswanathan K, Tomiya N, Park J, Singh S, Lee YC, Palter K, Betenbaugh MJ (2006) Expression of a functional Drosophila melanogaster CMP-sialic acid synthetase. Differential localization of the Drosophila and human enzymes. J Biol Chem 281:15929-15940.

von Gersdorff H, Schneggenburger R, Weis S, Neher E (1997) Presynaptic depression at a calyx synapse: the small contribution of metabotropic glutamate receptors. J Neurosci 17:8137-8146.

Wagh DA, Rasse TM, Asan E, Hofbauer A, Schwenkert I, Dürrbeck H, Buchner S, Dabauvalle MC, Schmidt M, Qin G, Wichmann C, Kittel R, Sigrist SJ, Buchner E (2006) Bruchpilot, a protein with homology to ELKS/CAST, is required for structural integrity and function of synaptic active zones in Drosophila. Neuron 49:833-844.

Wairkar YP, Fradkin LG, Noordermeer JN, DiAntonio A (2008) Synaptic defects in a Drosophila model of congenital muscular dystrophy. J Neurosci 28:3781-3789.

Wang JW, Sylwester AW, Reed D, Wu DA, Soll DR, Wu CF (1997) Morphometric description of the wandering behavior in Drosophila larvae: aberrant locomotion in $\mathrm{Na}^{+}$and $\mathrm{K}^{+}$channel mutants revealed by computer-assisted motion analysis. J Neurogenet 11:231-254.

Zhong Y, Wu CF (2004) Neuronal activity and adenylyl cyclase in environment-dependent plasticity of axonal outgrowth in Drosophila. J Neurosci 24:1439-1445.

Zuber C, Lackie PM, Catterall WA, Roth J (1992) Polysialic acid is associated with sodium channels and the neural cell adhesion molecule N-CAM in adult rat brain. J Biol Chem 267:9965-9971.

Zucker RS, Regehr WG (2002) Short-term synaptic plasticity. Annu Rev Physiol 64:355-405. 